\title{
Contrast-Induced Nephropathy in Patients Undergoing Elective Coronary Angiography: Incidence and Risk
}

\section{Factors}

\author{
Arsalan Salari, Fardin Mirbolouk, Bijan Shad, Mahboobeh Gholipour, Tolou Hasandokht, Amin Karimi, Azam \\ Nourisaeed and Samaneh Habibnejad \\ Guilan Interventional Cardiovascular Research Center, Guilan University of Medical Sciences, Rasht, Iran
}

\begin{abstract}
Contrast-Induced Nephropathy (CIN) is a considerable complication in cardiac procedures. Several conditions for CIN have been identified after Coronary Angiography (CA). The purpose of this study was to assess the incidence and clinical predictors of CIN $24 \mathrm{~h}$ after Coronary Angiography. A total of 1,137 consecutive patients with coronary artery syndrome undergoing CA were prospectively enrolled the study. Serum creatinine $(\mathrm{Cr})$ at baseline and $24 \mathrm{~h}$ after CA, as well as demographic and clinical characteristics of patients were measured. Contrast-induced nephropathy was defined as a rise in $\mathrm{Cr} \_0.3 \mathrm{mg} / \mathrm{dL}$ after $\mathrm{CA}$. Univariable and multivariable logistic regression analysis were performed to identify independent predictors of CIN. The overall incidence rate was 56 (4.9\%) in total study population. In multivariate analysis, baseline $\mathrm{Cr}>1.5$ (odds ratio [OR] 4.8, 95\% confidence interval [CI] 1.04 to $8.3 ; P<0.001$ ), Contrast volume $>100 \mathrm{mmL}$ (OR 3.4, 95\% CI 0.7 to $8.1 ; P<0.002$ ), Baseline GFR $<30$ (OR 14.2, 95\% CI 8-2; $P<0.000$ ); Baseline GFR 30-60 (OR 8.7, 95\% CI $2.313 .8 ; P<0.000$ ) were predictors for CIN. CIN was more frequent in older patients, with higher serum creatinine level and Grater usage of contrast media and diuretic. $\mathrm{N}$-acetylcysteine (NAC) and hydration cannot prevent the occurrence of CIN.
\end{abstract}

Key words: Contrast nephropathy, coronary angiography, contrast media.

\section{Introduction}

Acute renal failure (ARF) after coronary angiography is often attributed to Contrast-induced nephropathy (CIN) [1, 2]. Recent evidence suggests that CIN is associated with longer hospitalization [3], permanent renal failure $[1,2]$ and increased mortality [4-6]. Appropriate identification of at risk patients may reduce the duration of hospitalization and morbidity after CA [7]. Several risk factors are attributed to CIN, including: advancing age, volume of contrast medium, previous chronic renal failure, diabetes, reduced left ventricular systolic function, concomitant use of nephrotoxic drugs, and periprocedural hemodynamic instability [8-10]. Special conditions in cardiac procedures may

Corresponding author: Bijan Shad, associate professor, research field: general cardiology. predispose renal injury like predominant vascular atherosclerosis and hypotension [7]. Several studies assessed individual risk factors and cumulative effect of these risk factors on renal function in patients with acute myocardial infarction (AMI) $[1,10,11]$. Some studies assess the probability of renal insufficiency after PCI with the development of risk score models [10-12]. Although, the results of the various study were different and every study suggested different factors influence on occurrence of CIN. Monthly, a large number of coronary angiography have been conducted in referral heart centers of Guilan University of Medical Sciences, so, determining the frequency of CIN and its related factors seems to be necessary.

The purpose of the present prospective study was to determine the incidence of $\mathrm{CIN}$ and associated demographic and clinical factors for developing CIN 
after CA in patients with acute coronary syndrome in university teaching hospital of Guilan province, north of Iran.

\section{Patients and Methods}

\subsection{Study Population and Design}

Between September, 2013, and August, 2014, we enrolled all patients with coronary artery syndrome admitted to $\mathrm{Dr}$ Heshmat hospital, the referral cardiovascular hospital of Rash, Guilan province of Iran who were treated with elective CA. Patients with GFR $<30$ and candidate for dialysis as well as history of drug induced nephrotoxicity within the past two weeks such as non steroidal anti inflammatory drugs (NSAID), angiotensin receptor blocker (ARB), angiotensin converting enzyme inhibitor (ACEI) and diuretic were excluded. Diabetic patients had been discontinued metformin treatment two days before CA. Variables included demographic parameters, a detailed history of cardiovascular disease and co-morbidities, procedural details (dose of contrast medium used and prophylactic measures to prevent the development of CIN for each patient) were recorded. The decision to implement renal prophylactic measures as well as the type of these measures ( $\mathrm{N}$-acetylcysteine and hydration) was left to the discretion of the physicians caring for the patient.

Study design was approved by the ethics committee of the Interventional cardiovascular research center (ICRC) of Guilan University of Medical Science (GUMS) (Project number: 92040883), and written informed consent (per the Helsinki declaration) was obtained from each participant.

\subsection{Coronary Angiography}

Coronary Angiography was performed by an interventional team, according to standard clinical practice [13]. Type of contrast agent and contrast dose, angiography technique, and supportive pharmacologic therapies were based on the decision of the interventional cardiologist.

\subsection{Outcome and Data Measurements}

The study outcome was contrast-induced nephropathy, defined as an absolute serum creatinine increase $0.3 \mathrm{mg} / \mathrm{dL}$ or a relative increase in serum creatinine $50 \%$ that occurred within $24 \mathrm{~h}$ after coronary angiography [14]. The frequency of CIN was calculated using pre- and Post-procedural serum creatinine measurements after $24 \mathrm{~h}$. Preprocedural serum creatinine level and the glucose level were obtained closest to the time of coronary angiography during hospitalization. Creatinine clearance was calculated by applying the Cockcroft-Gault formula to the $\mathrm{Cr}$ [15].

\subsection{Statistical Analysis}

Results are reported as the mean value (SD) for continuous data and as absolute values and percentages for categorical data. Frequency of CIN was calculated in the entire sample. Comparison of continuous variables was compared by means of $t$ test. Chi-square tests were used for comparison of categorical variables. Multivariable logistic regression models were subsequently developed to evaluate the association between patient's condition and CIN. Patient characteristics and other risk factors identified in univariable logistic regression as predictors of CIN (at a level of significance of $P<0.05$ ), were entered into the models. Risk factors included demographic factors, comorbidities, and laboratory values, medications during hospitalization, contrast volume and preprocedural GFR. All comparisons were carried out on a two-tailed basis. Statistical analysis was carried out with the SPSS (version 16) and $P<0.05$ was considered statistically significant. Ninety-five percent significant intervals (CI) for the proportions were calculated.

\section{Results}

\subsection{Baseline Characteristics and Incidence of CIN}

A total of 1,137 patients (633 men, 504 women; mean age $59.5 \pm 1.5$ years) were included in this study. 
Diabetes and hypertension were present in 339 (29.8\%) and 458 patients (40.3) respectively. The mean creatinine for all study patients at baseline was 0.98 (0.3) $\mathrm{mg} / \mathrm{dL}$. More than fifty percent of patients had reported serum creatinine was greater than mean. Overall, 56 patients $(4.9 \%)$ developed CIN after coronary angiography. Mean creatinine in patients with CIN increased statistically significant from $1.2 \pm$ $0.9 \mathrm{mg} / \mathrm{dL}$ at baseline to $1.8 \pm 1.07 \mathrm{mg} / \mathrm{dL}$, while patients without CIN experienced no significant change in serum creatinine (baseline: $0.9 \pm 0.24$ $\mathrm{mg} / \mathrm{dL}$; post-PCI: $1.01 \pm 0.23 \mathrm{mg} / \mathrm{dL}$ ). The mean volume of contrast material administered was $103 \pm$ $30 \mathrm{~mL}$.

Table 1 shows the baseline clinical and procedural characteristics of patients who developed CIN and of those who did not present this complication after CA. Patients developing CIN were older, higher baseline $\mathrm{Cr}$ value. In addition, they received a higher volume of the contrast agent during PCI than patients without CIN. When creatinine clearance was estimated, 13 (1.1\%) and 165 (14.5) of the 1,137 patients had renal insufficiency $(<30 \mathrm{~mL} / \mathrm{min}$ and $30-60$, respectively). Of 13 patients with GFR $<30,3(23.1 \%)$ and of the 165 patients with GFR 30-60 mL/min, 13 (7.9\%) developed CIN. In contrast, of the 959 patients with a baseline GFR value $>60 \mathrm{~mL} / \mathrm{min}$, only $40(4.2 \%)$ developed CIN after PCI $(P<0.001)$. The medications taken by both groups were similar with the exception of diuretics, which were more common in the CIN group. Presence of hypertension, heart failure and diabetes mellitus were similar in both groups. Approximately $15.5 \%$ of patients received hydration with normal saline. Of them, 16 (9\%) developed CIN which was similar in cases without baseline hydration $45 / 959$ (4.7\%) $(P=0.45)$. No severe renal failure after intervention was seen in the study participants.

\subsection{Univariable Analysis}

Variables associated with $\mathrm{CIN}$ at $48 \mathrm{~h}$ inunivariable logistic regression models are shown in table 2. A total of 16 demographic, clinical, angiographic variables were studied. The five significantly associated variables included age $>60$ years (OR 1.2, 95\% CI 0.2-7.3; $P<$ 0.001 ), baseline creatinine levels $\geq 1.5 \mathrm{mg} / \mathrm{dL}$ (OR 3.32, 95\% CI 1.2-8.8; $P<0.017$ ), pre-existing renal insufficiency: GFR $<30$ as well as GFR $30=60$, (OR $6.8,95 \%$ CI 1.8-26; $P<0.004$, OR $1.9,95 \%$ CI 1.02-3.7; $P<0.04$, respectively), volume contrast use $\geq 100 \mathrm{~mL}$ (OR 1.6, 95\% CI 0.98-0.99; $P<0.01$ ), and diuretics (OR 1.4, 95\% CI 0.2-0.8; $P<0.01$ ).

In multivariate analysis, the following variables remained significant independent correlates of CIN: baseline $\mathrm{Cr}>1.5$ (odds ratio [OR] 4.8, 95\% confidence interval [CI] 1.04 to $8.3 ; P<0.001$ ), Contrast $>100 \mathrm{mmL}$ (OR 3.4, 95\% CI 0.7 to $8.1 ; P<$ 0.002), Baseline GFR $<30$ (OR 14.2, 95\% CI 8-2; $P<$ 0.000); Baseline GFR 30-60 (OR 8.7, 95\% CI 2.3 13.8; $P<0.000)$.

\section{Discussion}

We prospectively collected characteristics of a large of patients who underwent Coronary Angiography to assess the incidence of the CIN and its associated factors. CIN occurred in $4.9 \%$ of our study population at $24 \mathrm{~h}$ post CA. Gaetano M. De Ferrari et al showed the frequency of CIN after primary PCI in ST-segment elevation myocardial infarction (STEMI) was 5.6\% [16]. Some studies showed higher incidences of CIN than our study like $10.2 \%-19 \%[1,13,17,18]$ and some were similar $[16,19]$. In our study, baseline creatinine levels in patients were experienced CIN were higher than patients without CIN. In fact patient with pre-existing renal insufficiency, especially patients with GFR $<30,5$ times more reported CIN than cases with GFR $>60$. The odds of CIN in this group of study population about 14 times are higher than cases with GFR $>60$. This is in line with the results of several studies that established our findings [20, 21]. Peter A. McCullough [22] in a review article mentioned that reduced glomerular filtration at 
Table 1 Baseline and clinical characteristics of the study patients.

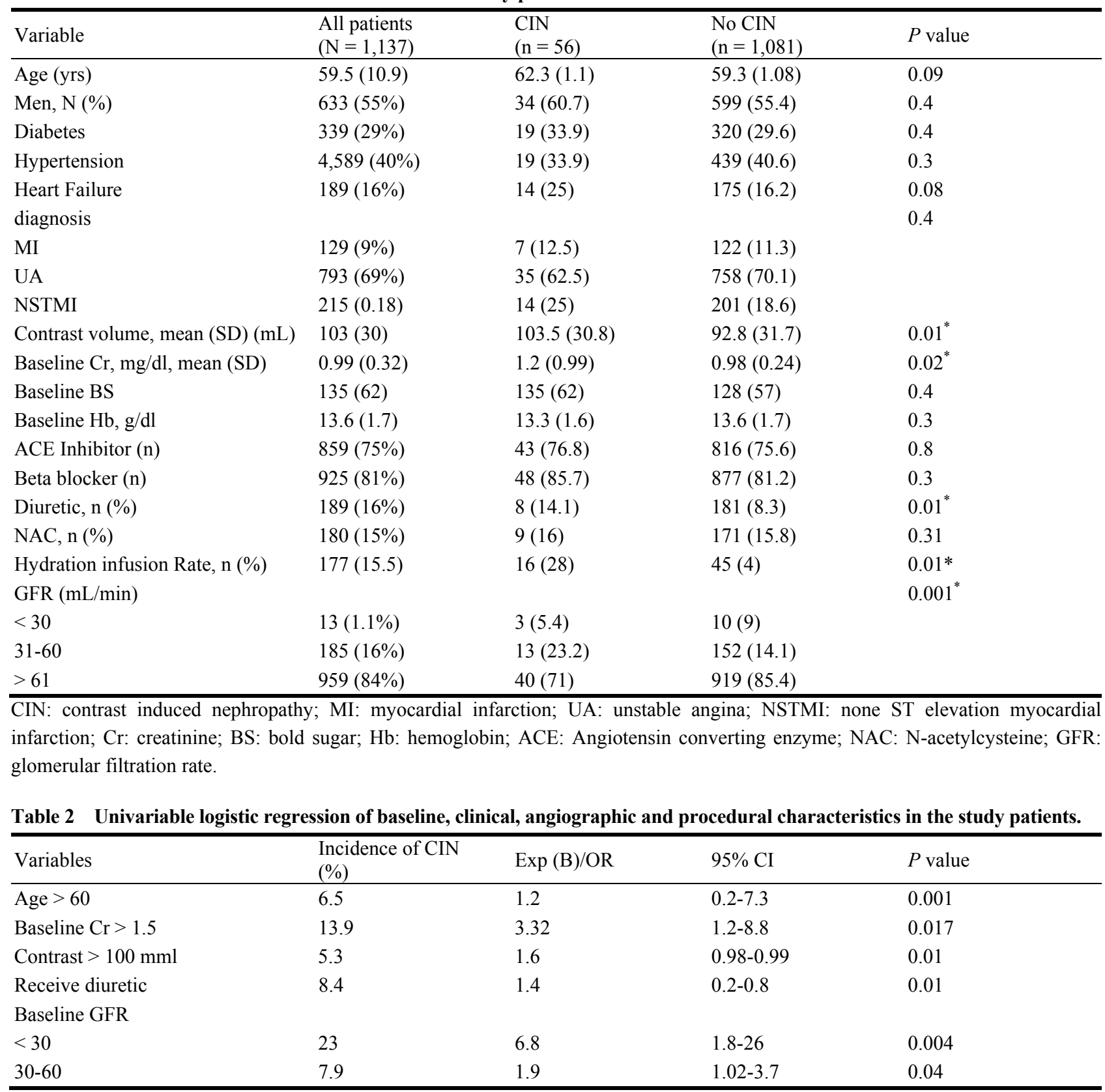

CIN: contrast induced nephropathy; OR: odds ratio; CI: confidence interval; GFR: glomerular filtration rate.

Table 3 Multivariable logistic regression of variables for CIN in the study patients.

\begin{tabular}{lllll}
\hline Variables & $\begin{array}{l}\text { Model coefficient } \\
\text { (b value) }\end{array}$ & OR & $95 \%$ CI & $P$ value \\
\hline Age $>65$ & 2.1 & 1.4 & $0.09-6.9$ & 0.021 \\
Baseline Cr $>1.5$ & 1.7 & 4.8 & $1.04-8.3$ & 0.001 \\
Contrast $>100 \mathrm{mml}$ & 1.3 & 3.4 & $0.7-8.1$ & 0.002 \\
Baseline GFR & & & & 0.000 \\
$<30$ & 4.9 & 14.2 & $8-21$ & 0.000 \\
$30-60$ & 2.9 & 8.7 & $2.3-13.8$ & \\
\hline
\end{tabular}

CIN: contrast induced nephropathy; OR: odds ratio; CI: confidence interval; Baseline GFR: glomerular filtration rate. 
baseline (GFR $<60 \mathrm{~mL} / \mathrm{min}$ ) is the single most important risk predictor for CIN, and should be a concerned for preventive measures. Similarly, Michael W et al. [23] designed a prospective study to evaluated incidence and risk factors of CIN after cardiac catheterization in elderly patients. They founded high level of creatinine at baseline an independent risk factors for renal dysfunction.

According to multivariate logistic regression analysis, we detect the odds of CIN $24 \mathrm{~h}$ after CA in cases with baseline creatinine $>1.5$ was approximately 5 times greater than in the study population with $\mathrm{Cr}<1.5$.

Our study revealed that old age and increased volume of contrast medium may enhance the occurrence of CIN after Coronary Angiography, in agreement with previously published evidence [10, 12, 13, 24]. The present study failed to illustrate the predictive effect of some factors including; diabetes, hypertension, heart failure anemia, and treatment measures ( $\mathrm{N}$-acytelcysteine use and hydration). Whereas, Mehran and colleague [10] as well as Bartholomew et al. [11], identified variables such as older age, chronic heart failure, diabetes, contrast volume, and anemia that were associated with increased risk of CIN. However, Dimitrios Tziakas et al proposed a risk score model for contrast-induced nephropathy prediction after PCI, with good discriminating power (c-statistic 0.759) and excellent calibration (calibration slope 0.91) regarding Pre-existing renal disease, metformin, history of previous PCI, peripheral arterial disease, and contrast volume $\geq 300 \mathrm{~mL}$. Treatment with $\mathrm{N}$-acytelcysteine seems to have no protective effect on CIN after cardiac catheterization $[10,11]$. Two meta-analyses showed protective effect of NAC individually with higher doses and intravenous use [25, 26], whereas, Otavio Berwanger in a large randomized trial, found that acetylcysteine does not reduce the risk of contrast-induced nephropathy in high risk or low risk patients undergoing coronary and peripheral vascular angiography [27].

Our study has some limitations. First, we did not assess a several characteristics that affect on end points such as previous history of $\mathrm{CA}$, the number of involved vessels. Second, since we used creatinine level as indicator of renal insufficiency, the low incidence of CIN were reported than other study. While, several study proposed cystatin $\mathrm{C}$ are more reliable for detecting contrast-induced nephropathy [28-31]. On the other hands, the median volume of contrast used was $100 \mathrm{~mL}$, whereas majority of previous studies use greater volume of contrast media like $300 \mathrm{~mL}[4,13,31,32]$. Furthermore, there was no follow-upprogram after $24 \mathrm{~h}$ to assess the incidence of CIN.

In conclusion, our study showed that the most important factors affected the incidence of CIN after CA were old age, pre-existing renal insufficiency, diuretic medication use and the volume of contrast media. According to our study the prophylactic measures did not result in a lower incidence of contrast-induced nephropathy. Hence, improving clinical decisionmaking may prevent unnecessary procedure and complication.

Disclosure statement: The authors report no financial relationships or conflicts of interest regarding the content herein.

\section{References}

[1] Marenzi, G., Assanelli, E., Campodonico, J., Lauri, G., Marana, I., De Metrio, M., Moltrasio, M., Grazi, M., Rubino, M., Veglia, F., Fabbiocchi, F., and Bartorelli, A. L. 2009. "Contrast Volume During Primary Percutaneous Coronary Intervention and Subsequent Contrast-Induced Nephropathy and Mortality." Annals of Internal Medicine 150 (3): 170-7.

[2] Assali, A. R., Brosh, D., Ben-Dor, I., Solodky, A., Fuchs, S., Teplitsky, I., and Kornowski, R. 2007. "The Impact of Renal Insufficiency on Patients Outcomes in Emergent Angioplasty for Acute Myocardial Infarction." Catheterization and Cardiovascular Interventions 69 (3): 395-400.

[3] Aronow, H. D., Peyser, P. A., Eagle, K. A., Bates, E. R., Werns, S. W., Russman, P. L., Crum, M. A., Harris, K., 
and Moscucci, M. 2001. "Predictors of Length of Stay after Coronary Stenting." American Heart Journal 142 (5): 799-805.

[4] Bouzas-Mosquera, A., Vázquez-Rodríguez, J. M., Calviño-Santos, R., Peteiro-Vázquez, J., Flores-Ríos, X., Marzoa-Rivas, R., Piñón-Esteban, P., Aldama-López, G., Salgado-Fernández, J., and Vázquez-González, N. 2007. "Contrast-induced Nephropathy and Acute Renal Failure following Emergent Cardiac Catheterization: Incidence, Risk Factors and Prognosis." Revista Española de Cardiología 60 (10): 1026-34.

[5] McCullough, P. A. 2006. CIN Consensus Working Panel: Executive Summary.

[6] James, M. T., Samuel, S. M., Manning, M. A., Tonelli, M., Ghali, W. A., Faris, P., Knudtson, M. L., Pannu, N., and Hemmelgarn, B. R. 2013. "Contrast-Induced Acute Kidney Injury and Risk of Adverse Clinical Outcomes after Coronary Angiography A Systematic Review and Meta-Analysis." Circulation: Cardiovascular Interventions 6 (1): 37-43.

[7] Barrett, B. J., and Parfrey, P. S. 2006. "Preventing Nephropathy Induced by Contrast Medium." New England Journal of Medicine 354 (4): 379-86.

[8] Goldenberg, I., and Matetzky, S. 2005. "Nephropathy Induced by Contrast Media: Pathogenesis, Risk Factors and Preventive Strategies." Canadian Medical Association Journal 172 (11): 1461-71.

[9] Dangas, G., Iakovou, I., Nikolsky, E., Aymong, E. D., Mintz, G. S., Kipshidze, N. N., Lansky, A. J., Moussa, I., Stone, G. W., and Moses, J. W. 2005. "Contrast-induced Nephropathy after Percutaneous Coronary Interventions in Relation to Chronic Kidney Disease and Hemodynamic Variables." The American Journal of Cardiology 95 (1): 13-9.

[10] Mehran, R., Aymong, E. D., Nikolsky, E., Lasic, Z., Iakovou, I., Fahy, M., Mintz, G. S., Lansky, A. J., Moses, J. W., and Stone, G. W. 2004. "A Simple Risk Score for Prediction of Contrast-induced Nephropathy after Percutaneous Coronary Intervention Development and Initial Validation." Journal of the American College of Cardiology 44 (7): 1393-9.

[11] Bartholomew, B. A., Harjai, K. J., Dukkipati, S., Boura, J. A., Yerkey, M. W., Glazier, S., Grines, C. L., and O'Neill, W. W. 2004. "Impact of Nephropathy after Percutaneous Coronary Intervention and a Method for Risk Stratification." The American Journal of Cardiology 93 (12): 1515-9.

[12] Marenzi, G., Lauri, G., Assanelli, E., Campodonico, J., De Metrio, M., Marana, I., Grazi, M., Veglia, F., and Bartorelli, A. L. 2004. "Contrast-induced Nephropathy in Patients Undergoing Primary Angioplasty for Acute
Myocardial Infarction." Journal of the American College of Cardiology 44 (9): 1780-5.

[13] Tziakas, D., Chalikias, G., Stakos, D., Apostolakis, S., Adina, T., Kikas, P., Alexoudis, A., Passadakis, P., Thodis, E., and Vargemezis, V. 2013. "Development of an Easily Applicable Risk Score Model for Contrast-induced Nephropathy Prediction after Percutaneous Coronary Intervention: a Novel Approach Tailored to Current Practice." International Journal of Cardiology 163 (1): 46-55.

[14] Mehta, R. L., Kellum, J. A., Shah, S. V., Molitoris, B. A., Ronco, C., Warnock, D. G., and Levin, A. 2007. “Acute Kidney Injury Network: Report of an Initiative to Improve Outcomes in Acute Kidney Injury." Critical Care 11 (2): R31.

[15] Cockcroft, D. W., and Gault, M. H. 1976. "Prediction of Creatinine Clearance from Serum Creatinine." Nephron 16 (1): 31-41.

[16] De Ferrari, G. M., Somaschini, A., Cornara, S., Crimi, G., Baldo, A., Pavesi, C., Pica, S., Ferlini, M., Camporotondo, R., and Gnecchi, M. 2014. "REAL PROGNOSTIC ROLE OF CONTRAST-INDUCED NEPHROPATHY IN PATIENTS WITH ST-SEGMENT ELEVATION MYOCARDIAL INFARCTION UNDERGOING PRIMARY PERCUTANEOUS CORONARY INTERVENTION." Journal of the American College of Cardiology 63 (12_S).

[17] Maioli, M., Toso, A., Leoncini, M., Gallopin, M., Musilli, N., and Bellandi, F. 2012. "Persistent Renal Damage after Contrast-induced Acute Kidney Injury: Incidence, Evolution, Risk Factors and Prognosis." Circulation CIRCULATIONAHA. 111.085290.

[18] Nikolsky, E., Mehran, R., Lasic, Z., Mintz, G. S., Lansky, A. J., Na, Y., Pocock, S., Negoita, M., Moussa, I., and Stone, G. W. 2005. "Low Hematocrit Predicts Contrast-induced Nephropathy after Percutaneous Coronary Interventions." Kidney International 67 (2): 706-13.

[19] Aspelin, P., Aubry, P., Fransson, S-G., Strasser, R., Willenbrock, R., and Berg, K. J. 2003. "Nephrotoxic Effects in High-risk Patients Undergoing Angiography." New England Journal of Medicine 348 (6): 491-9.

[20] Rihal, C. S., Textor, S. C., Grill, D. E., Berger, P. B., Ting, H. H., Best, P. J., Singh, M., Bell, M. R., Barsness, G. W., and Mathew, V. 2002. "Incidence and Prognostic Importance of Acute Renal Failure after Percutaneous Coronary Intervention." Circulation 105 (19): 2259-64.

[21] Rubenstein, M. H., Sheynberg, B. V., Harrell, L. C., Schunkert, H., Bazari, H., and Palacios, I. F. 2001. "Effectiveness of Adverse Events after Percutaneous Coronary Intervention in Patients with Mild versus 

Incidence and Risk Factors

Severe Renal Failure." The American Journal of Cardiology 87 (7): 856-60.

[22] McCullough, P. A. 2014. "Contrast-Induced Nephropathy: Definitions, Epidemiology, and Implications." Interventional Cardiology Clinics 3 (3): 357-62.

[23] Rich, M. W., and Crecelius, C. A. 1990. "Incidence, Risk Factors, and Clinical Course of Acute Renal Insufficiency after Cardiac Catheterization in Patients 70 Years of Age or Older: a Prospective Study." Archives of Internal Medicine 150 (6): 1237-42.

[24] Bertrand, M. E., Esplugas, E., Piessens, J., and Rasch, W. 2000. "Influence of a Nonionic, Iso-osmolar Contrast Medium (iodixanol) versus an Ionic, Low-osmolar Contrast Medium (ioxaglate) on Major Adverse Cardiac Events in Patients undergoing Percutaneous Transluminal Coronary Angioplasty a Multicenter, Randomized, Double-blind Study." Circulation 101 (2): 131-6.

[25] Trivedi, H., Daram, S., Szabo, A., Bartorelli, A. L., and Marenzi, G. 2009. "High-dose N-acetylcysteine for the Prevention of Contrast-induced Nephropathy." The American Journal of Medicine 122 (9): 874. e9-e15.

[26] Kelly, A. M., Dwamena, B., Cronin, P., Bernstein, S. J., and Carlos, R. C. 2008. "Meta-analysis: Effectiveness of Drugs for Preventing Contrast-induced Nephropathy." Annals of Internal Medicine 148 (4): 284-94.

[27] Investigators, A. 2011. "Acetylcysteine for Prevention of Renal Outcomes in Patients undergoing Coronary and Peripheral Vascular Angiography Main Results from the
Randomized Acetylcysteine for Contrast-Induced Nephropathy Trial (ACT)." Circulation 124 (11): 1250-9.

[28] Hoffmann, U., Fischereder, M., Krüger, B., Drobnik, W., and Krämer, B. K. 2004. "The Value of N-acetylcysteine in the Prevention of Radiocontrast Agent-induced Nephropathy Seems Questionable." Journal of the American Society of Nephrology 15 (2): 407-10.

[29] D'elia, J., Gleason, R., Alday, M., Malarick, C., Godley, K., Warram, J., Kaldany, A., and Weinrauch, L. 1982. "Nephrotoxicity from Angiographic Contrast Material: a Prospective Study." The American Journal of Medicine 72 (5): 719-25.

[30] Droppa, M., Desch, S., Blase, P., Eitel, I., Fuernau, G., Schuler, G., Adams, V., and Thiele, H. 2011. "Impact of $\mathrm{N}$-acetylcysteine on Contrast-induced Nephropathy Defined by Cystatin C in Patients with ST-elevation Myocardial Infarction undergoing Primary Angioplasty." Clinical Research in Cardiology 100 (11): 1037-43.

[31] Tanaga, K., Tarao, K., Nakamura, Y., Inoue, T., Jo, K., Ishikawa, T., and Miyazaki, A. 2012. "Percutaneous Coronary Intervention Causes Increase of Serum Cystatin C Concentration Even in the Patients with a Low Risk of Contrast-induced Nephropathy." Cardiovascular Intervention and Therapeutics 27 (3): 168-73.

[32] Aghasadeghi, K., and Akbari, V. 2008. "Comparison of $\mathrm{N}$-acetylcysteine and Ascorbic Acid in Prevention of Renal Dysfunction after Coronary Angioplasty." $J$. Renovasc. Dis. 6: 1-5. 